\title{
Immunohistochemical detection of prothymosin alpha in pituitary adenomas - a new marker of tumor recurrence?
}

\author{
Marek Pawlikowski and Katarzyna Winczyk
}

Department of Neuroendocrinology, Chair of Endocrinology, Medical University of Łódź, Łódź, Poland

\begin{abstract}
Forty pituitary adenomas were immunostained with an antibody raised against the C-terminal fragment (101-109) of human prothymosin alpha $(\mathrm{PT} \alpha)$. The strong positive immunostaining was found in the subpopulation of cell nuclei and intratumoral vessel walls, while the cytoplasm of adenoma cells was slightly immunopositive. The significantly higher percentage of PT $\alpha$-positive cell nuclei was found in recurrent pituitary adenomas as compared with primary tumors. However, there was no correlation between the percentage of PT $\alpha$-positive cell nuclei and Ki-67 indices. Gonadotropinomas were characterized by higher nuclear PT $\alpha$ expression in comparison to other pituitary adenomas, which is probably linked with the high recurrence rate of these tumors. It is suggested that PT $\alpha$ immunostaining may be helpful in predicting the pituitary tumor recurrence. However, this conclusion needs to be confirmed in further prospective studies. Moreover, PT $\alpha$ may be also useful as an immunohistochemical marker of the intratumoral microvasculature.
\end{abstract}

Key words: pituitary adenomas, prothymosin alpha, Ki-67, prognostic factors

\section{Introduction}

Prothymosin alpha $(\mathrm{PT} \alpha)$ was originally isolated from thymus and initially considered as a thymic hormone. It is a peptide composed in humans from 111 amino acids and it shows a wide distribution in different tissues and organs [1]. Its physiological role remains obscure. However, because of its dominant intra-nuclear localization a role of PT $\alpha$ in the control of cell proliferation and/or of apoptosis was suggested $[2,3]$. In some recent studies an overexpression of PT $\alpha$ was found in malignant tumors, like gastric [4], prostate $[5,6]$ and thyroid cancers $[7,8]$. The quoted authors suggest that PT $\alpha$ may be useful as a marker of malignancy. Although pituitary adenomas are considered as benign tumors, they markedly differ from case to case in their aggressiveness, related to the ability to invade the adjacent structures and to the growth potential. To our knowledge, there are no data on PT $\alpha$ expression in pituitary adenomas except the preliminary study published by one of us several years ago $[9,10]$. Recently, a group from Greece developed and characterized an antibody against the C-terminal frag-

Correspondence: M. Pawlikowski, MD, Chair of Endocrinology, Medical University of Łódź, Sterling str.3, 91-425 Łódź, Poland; e-mail: marek.pawlikowski@umed.lodz.pl ment 101-109 of human PT $\alpha$, suitable for immunohistochemistry [6]. The present study reports on the immunohistochemical detection of PT $\alpha$ in different subtypes of pituitary adenomas using this antibody.

\section{Material and methods}

The study was performed using the archival material of 40 pituitary adenomas stored as paraffin blocks. This material included 6 cases of acromegaly, 3 cases of prolactinoma, 24 cases of clinically non-functioning pituitary adenomas (CNFPA, including 8 adenomas expressing solely gonadotrophins or free $\alpha$-subunit), 7 cases of Cushing's disease (including one case of Nelson's syndrome). The paraffin sections of the tumors were immunostained with antibody against the C-terminal fragment (101-109) of prothymosin alpha (kindly received from Dr Evangelia Livaniou, Institute of Radioisotopes and Radiodiagnostic products, National Science for Scientific Research "Demokritos", Athens, Greece) or with anti-Ki-67 antigen (MIB-1) antibody (Dako-Cytomation, Denmark). The primary antibodies were applied in the working solution 1:100. The control slides were stained with the omission of the primary antibody. The hormonal phenotype of the investigated pituitary adenomas has been established previously by means of immunostaining with antibodies to pituitary hormones (GH, PRL, FSH, LH, TSH, ACTH and $\alpha$-subunit). The visualization of reactions was done by means of streptavidin-biotin-peroxidase technique with use of StreptABC/HRP kit (Dako-Cytomation) and 3,3'-diaminobenzidine as chromogen. The number of PT $\alpha$-immunopositive and Ki-67-immunopositive cell nuclei was estimated in 500 randomly counted cell nuclei and expressed as percentage (\%). 

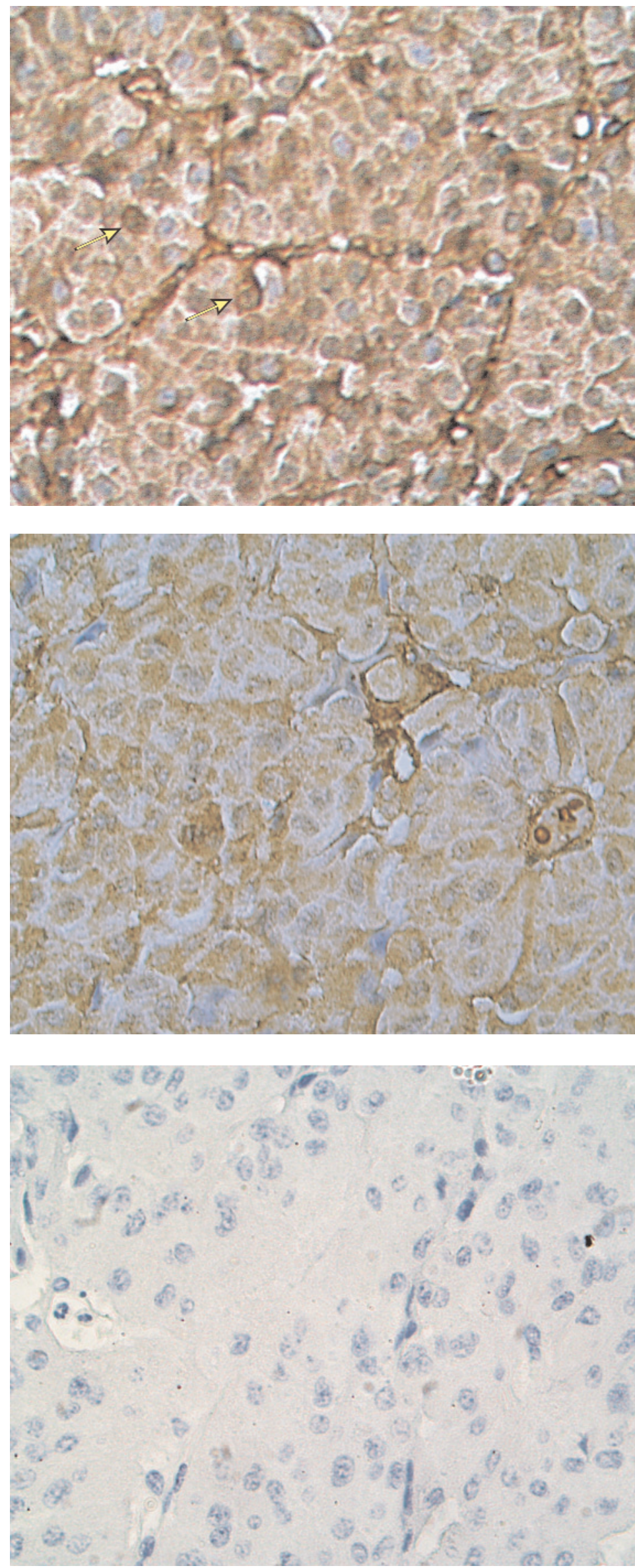

Fig. 1. Immunostaining with anti-PT $\alpha$ antibody in the clinically non-functioning, ACTH-immunopositive recurrent pituitary adenoma (second recurrence) of the male patient - ZL. Arrows indicate PT $\alpha$-immunopositive (brown) cell nuclei. The PT $\alpha$-immunopositive microvessels (dark brown) can also be seen (original magnification $\times 400$ ).

Fig. 2. Immunostaining with anti-PT $\alpha$ antibody in the clinically non-functioning, $\mathrm{ACTH}$-immunopositive pituitary adenoma of the male patient WW. The cell nuclei are mostly immunonegative (original magnification $\times 400$ ).
Fig. 3. The same tumor as on Fig. 2. The negative control (without the primary antibody) (original magnification $\times 400)$. 
The numeric data were analyzed statistically using ANOVA followed by LSD test; statistical significance was at level $\mathrm{p}<0.05$.

\section{Results}

The strong immunostaining with PT $\alpha$ antibody was found in a subpopulation of tumor cell nuclei and in the endothelium of intratumoral blood vessels. Less intensive immunostaining was also noticed in adenoma cells cytoplasm (Fig. 1, 2). No staining was found in control slides, where the primary antibody was omitted (Fig. 3). The percentage of PT $\alpha$-immunopositive nuclei was clearly higher in recurrent adenomas than in primary tumors $(19.7 \pm 3.9 \%$ vs $3.5 \pm 0.8 \%$; mean \pm SEM, $p<0.001)$. However, there was no correlation between the frequency of PT $\alpha$-immunopositive nuclei and Ki-67 index (data not shown). Moreover, there was no significant difference in Ki-67 index between recurrent and primary adenomas $(2.59 \pm 0.96$ vs $4.03 \pm 1.4$, mean \pm SEM). The mean frequency of PT $\alpha$-immunopositive cell nuclei in dependence on the hormonal phenotype of adenomas is shown in Fig. 4. As it can be seen there, the highest percentage of PT $\alpha$ immunopositive subpopulation of cell nuclei occurred in gonadotropinomas. No differences were observed in cytoplasmic PT $\alpha$ immunostaing either between recurrent and non-recurrent tumors or in dependence of hormonal phenotype of the tumor, except the corticotropinomas in Cushing's disease, which demonstrate a slightly higher cytoplasmic expression.

\section{Discussion}

The preferential nuclear PT $\alpha$ immunostaining in pituitary adenomas is concordant with our earlier data [9] and compatible with the observations concerning the other tissues either normal or neoplastic [cited in the introduction]. The most important finding of the present study is showing that the nuclear expression of $\mathrm{PT} \alpha$ is significantly higher in recurrent adenomas in comparison to primary tumors. This observation corroborates with our earlier preliminary study $[9,10]$. Nuclear expression of PT $\alpha$ does not reveal the significant differences depending on different hormonal phenotypes of adenomas, except the significantly higher number of PT $\alpha$-immunopositive nuclei in gonadotropinomas. However, this result probably depends on the highest rate of recurrent tumors within this group. The risk of recurrence of the pituitary adenoma after surgery is complex and it depends on the time of diagnosis and skillfulness of the neurosurgeon, but also on the tumor aggressiveness. The latter includes the tumor invasiveness, which enables the total excision of the tumor, and the high proliferation potency. It was shown that recurrent pituitary adenomas exhibit higher levels of different proliferation

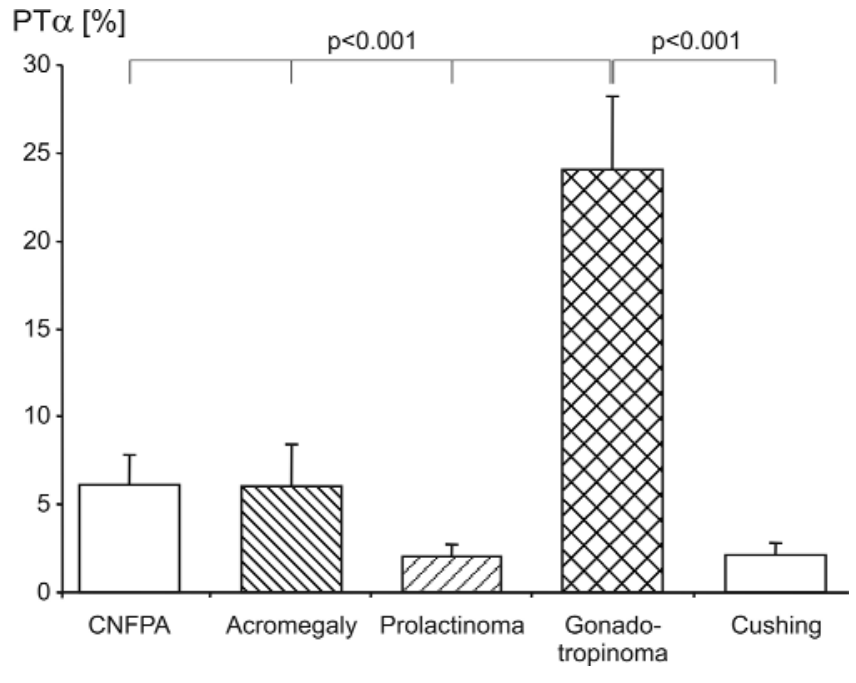

Fig. 4. Percentages of PT $\alpha$-immunopositive cell nuclei in dependence of the hormonal phenotype of pituitary adenomas. (CNFPA clinically non-functioning pituitary adenomas).

markers, like silver staining nuclear organizer regions - AgNOR, proliferating cells nuclear antigen - PCNA or Ki-67 than non-recurrent pituitary tumors [11-15]. However, according to other authors, Ki-67 index is not a predictor of tumor recurrence [16]. Turner and Wass [17] conclude that although there is a relationship in many pituitary tumors between Ki-67 index and their invasiveness and recurrence, the low Ki-67 index can be noticed in aggressive tumors and, in contrast, the high proliferation index may occur in the indolent adenomas. In the present study, we did not find a significant difference in Ki-67 index between recurrent and primary tumors. These data taken together suggest that the value of $\mathrm{Ki}-67$ labeling alone is limited as prognostic factor. Possibly, PT $\alpha$ immunostaining may be helpful also in predicting the pituitary tumor recurrence. However, this conclusion needs to be confirmed in further prospective studies. Another sites of the strong PT $\alpha$ immunostaining are intratumoral microvessel walls. In the larger vessels it can be observed that the PT $\alpha$ immunostaining concerns mostly endothelium. It remains unclear whether endothelial cells are able to express intrinsically the large amounts of PT $\alpha$ or the peptide produced in tumoral cells preferentially bind to endothelium. Independently of these explanations, the strong immunostaining of PT $\alpha$ within the endothelium corroborates with the suggested pro-angiogenic role of this peptide [18]. Moreover, PT $\propto$ may be useful as an immunohistochemical marker of the intra-tumoral microvasculature.

Acknowledgements: The authors thank Dr Evangelia Livaniou from the Institute of Radioisotopes and Radiodiagnostic Products, National Science for Scientific Research "Demokritos", Athens, Greece) for a kind gift of anti-PT $\alpha$ antibody. We are also grateful to Professor Jolanta Kunert-Radek from the Department of Clini- 
cal Endocrinology and Professor Andrzej Radek from the Department of Neurosurgery and Surgery of Peripheral Nerves, Medical University of Łódź, for their help in collecting the material of pituitary adenomas and to Mrs Maria Jaranowska, Mrs Małgorzata Jędrzejewska and Mrs Anna Opłatowska for their skillful technical assistance. Paper was supported by Medical University of Łódź grant No 503-5084-1.

\section{References}

[ 1] Pineiro A, Cordero OJ, Noguiera M. Fifteen years of prothymosin alpha: contradictory past and new horizons. Peptides. 2000;21:1433-1446.

[2] Letsas KP, Frangou-Lazaridis M. Surfing on prothymosin alpha proliferation and anti-apoptotic properties. Neoplasma. 2006;53:92-96

[3] Sburlati AR, Manrow RE, Berger SL. Prothymosin alpha antisense oligomers inhibit myeloma cell division. Proc Natl Acad Sci USA. 1991;88:253-257.

[4] Leys CM, Nomura S, LaFleur BJ et al. Expression and prognostic significance of prothymosin-alpha and ERp57 in human gastric cancer. Surgery. 2007;141:41-50.

[5] Suzuki S, Takahashi S, Takeshita K et al. Expression of prothymosin alpha is correlated with development and progression in human prostatic cancers. Prostate. 2006;66: 463-469.

[ 6] Klimentzou P, Drougou A, Fehrenbacher B et al. Immunocytological and preliminary immunohistochemical studies of peothymnosin alpha, a human cancer-associated polypeptide, with a well-characterized polyclonal antibody. $J$ Histochem Cytochem. 2008;56:1023-1031.

[7] Letsas KP, Vartholomatos G, Tsepi C, Tsatsoulis A, FrangouLazaridis M. Fine needle aspiration biopsy RT-PCR expression analysis of prothymosin alpha and parathymosin in thyroid: novel proliferation markers? Neoplasma. 2007;54:5762.

[8] Letsas KP, Frangou-Lazaridis M, Skyrlas A, Tsatsoulis A, Malamou-Mitsi V. Transcription factor-mediated proliferation and apoptosis in benign and malignant thyroid lesions. Pathol Int. 2005;55:694-702.

[9] Pawlikowski M. Immunostaining of thymosin peptides in human pituitary adenomas. Int $J$ Thymol. 1999;7:607-611.

[10] Pawlikowski M. Immunohistochemical markers of pituitary tumor aggressiveness (in polish). Endokr Pol-Polish $J$ Endocrinol. 2002;53(suppl 1):238-242.

[11] Słowińska-Klencka D, Klencki M, Dedecjus M, Lewiński A, Pawlikowski M. Silver-staining nucleolar organizer region quantification in pituitary adenomas. Endocrine Pathol. 2000;11:173-178.

[12] Klencki M, Słowinska-Klencka D, Lewinski A, Pawlikowski M. Correlation between PCNA expression and AgNOR dots in pituitary adenomas. Endocr Pathol. 2001;12:163-169

[13] Hsu DW, Hakim F, Biller BM et al. Significance of proliferating cell nuclear antigen index in predicting pituitary tumor recurrence. J Neurosurg. 1993;78:753-761.

[14] Pawlikowski M, Gruszka A, Kurnatowska I, Winczyk K, Kunert-Radek J, Radek A Proliferating cell nuclear antigen (PCNA) expression in pituitary adenomas: relationship to the endocrine phenotype of adenoma. Folia Histochem Cytobiol. 2006;44:37-41.

[15] Paek KI, Kim SH, Song SH et al. Clinical significance of Ki67 labeling index in pituitary macroadenoma. J Korean Med Sci. 2005;20:489-494.

[16] Losa N, Franzin A, Mangili F et al. Proliferation index of nonfunctioning pituitary adenomas: correlation with clinical characteristics and long-term follow-up results. Neurosurgery. 2000;47:1313-1318.

[17] Turner HE, Wass JA. Are markers of proliferation valuable in the histological assessment of pituitary tumours? Pituitary. 1999;1:147-151.

[18] Grant DS, Kinsella JL, Kibbey MC et al. A matrigel induced gene involved in endothelial cell proliferation and angiogenesis. J Cell Sci. 1995; 108: 3683-3694.

Submitted: 5 June, 2009 Accepted after reviews: 5 November, 2009 